\title{
Neutrino Trident Production at the MINERvA Experiment
}

\author{
Jose Antonio Becerra, Sebastián Sánchez Falero, \\ Alberto Gago, Eric Endress \\ PONTIFICIA UNIVERSIDAD CATÓLICA DEL PERÚ
}

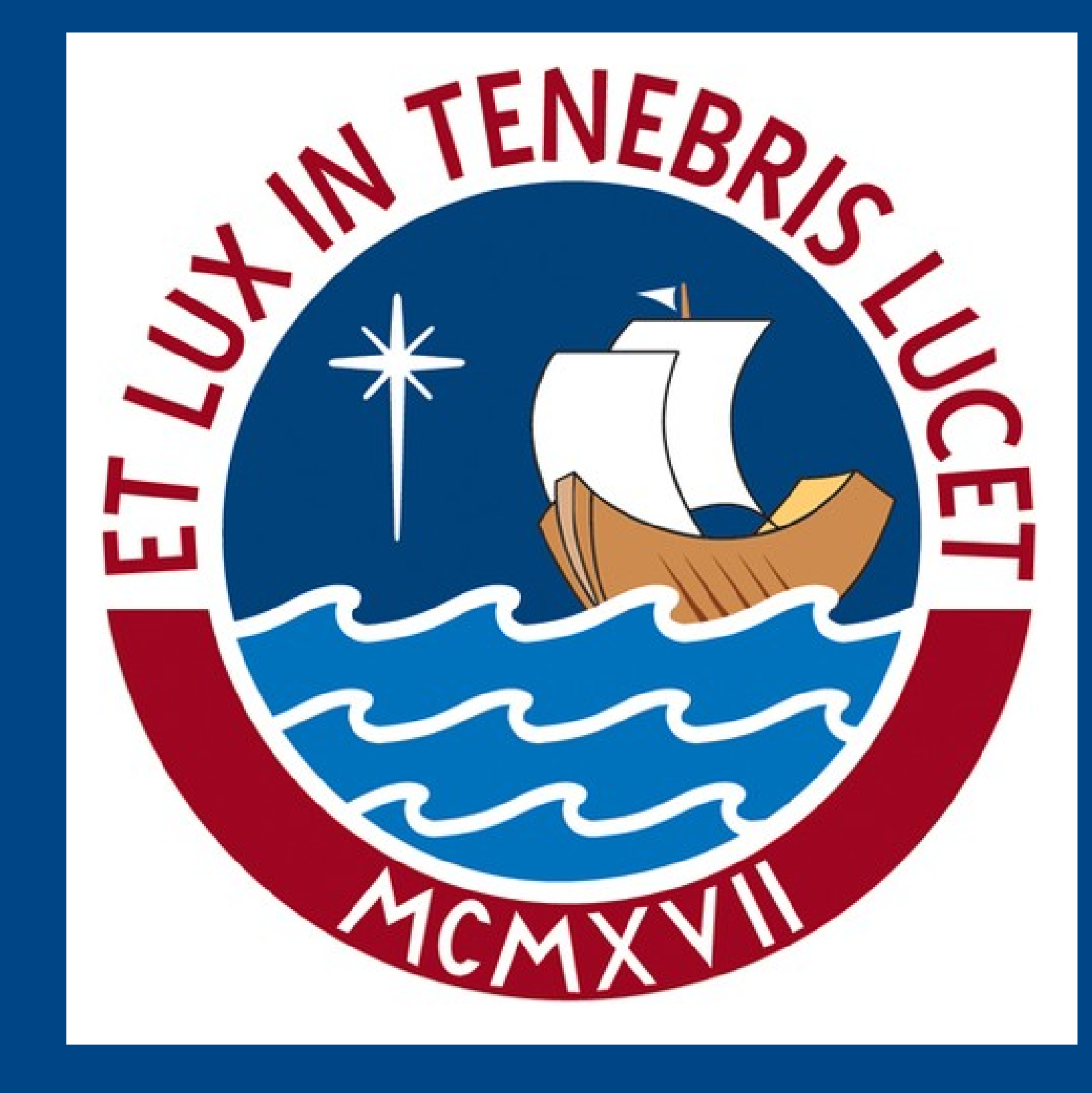

INTRODUCTION

- We performed a detailed simulation and optimized search of the signature of neutrino Trident events in a Minerva-like experiment,

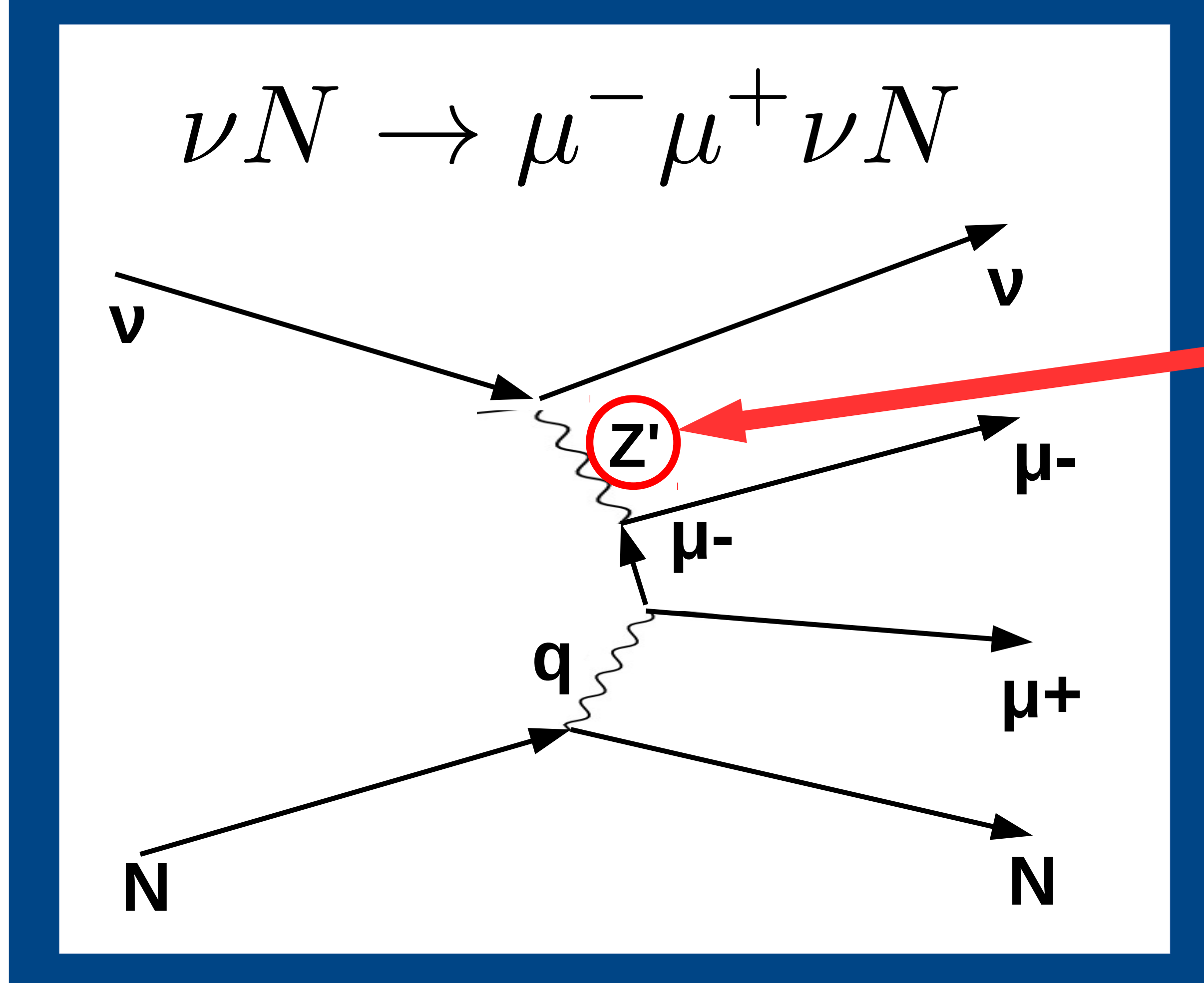

- Signal Definition:

1) Two oppositely charged muons.

2) Coherent interaction

( No hadronic shower)

- Why is it of interest?

It could be used to look for new $Z^{\prime}$ Bosons and test extensions of the Standard Model[2]

Figure 1: Trident Feynman Diagram

\section{SIMULATION FRAMEWORK}

We simulated events coming from the NuMI ME Beam[5].

- Signal: Produced with GENIE v.2.12.4 [3], modified to include the Trident production channel.

- Background:

- Problem: Not enough statistics for TMVA Analysis in the MINERvA ME Monte Carlo samples ( POT $=9.5 \mathrm{e} 20$ ).

- Solution: Events were produced in GENIE and propagated in a simplified version of the Minerva tracker region using GEANT4 v10.3.1

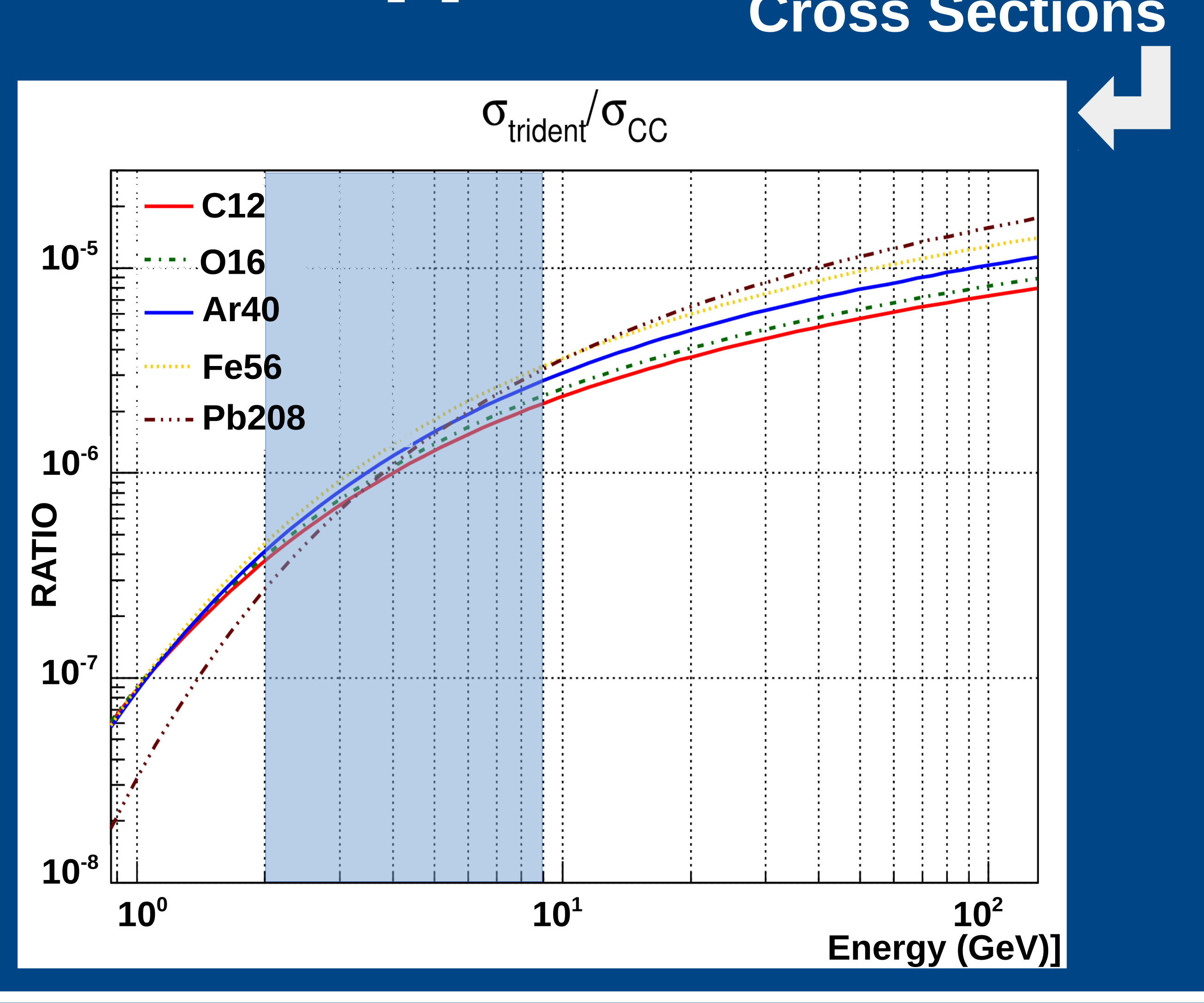

\section{ANALYSIS AND RESULTS}

Depending on Detector design, not all observables will be available!!

We study several scenarios for different combinations of observables

\begin{tabular}{ll} 
Obs & \multicolumn{1}{c}{ Description } \\
$Z_{\text {int }}$ & Longitudinal Vertex Position \\
$\Theta+$ & mu+ angle with beam axis \\
$\Theta-$ & mu- angle with beam axis \\
$\Theta_{+-}$ & Angle between mu- and mu+ \\
$E_{+-}$ & Energy difference between \\
$W_{+-}$ & Invariant Mass
\end{tabular}

Table 1: Observables considered
- Boosted Fisher:

toolkit for TMVA v.4.2.0 ROOT to train and test two methods[4]:

\section{- Boosted Decision Trees} Obs Description

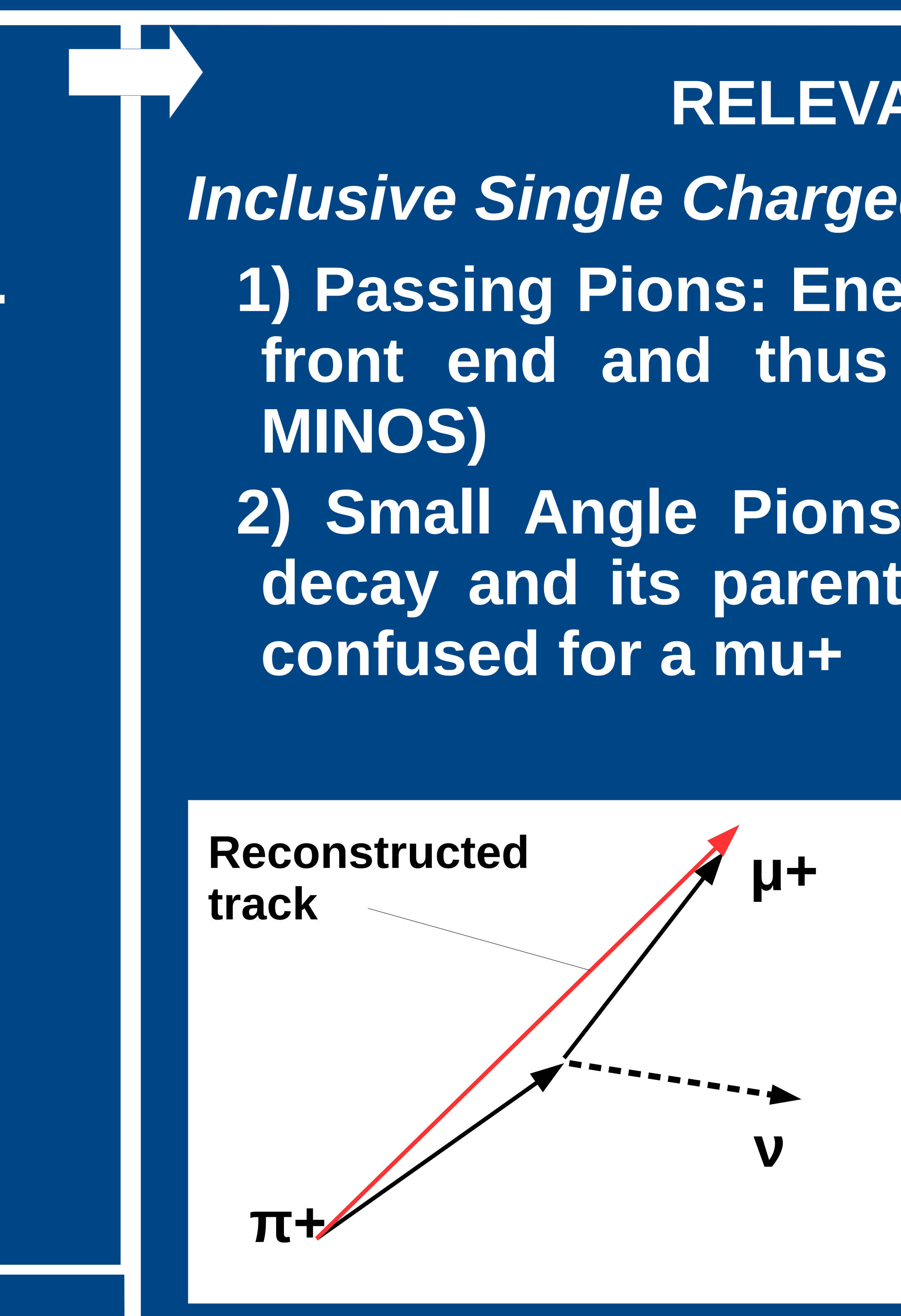

Figure 2: Small Angle Pions Background

\section{Charm Production}

1) Only two muons in Final State

2) No hadronic shower $\underline{\text { OR }}$

3) Events with neutrons in the final state.

Why? Neutrons are not always visible to the detector

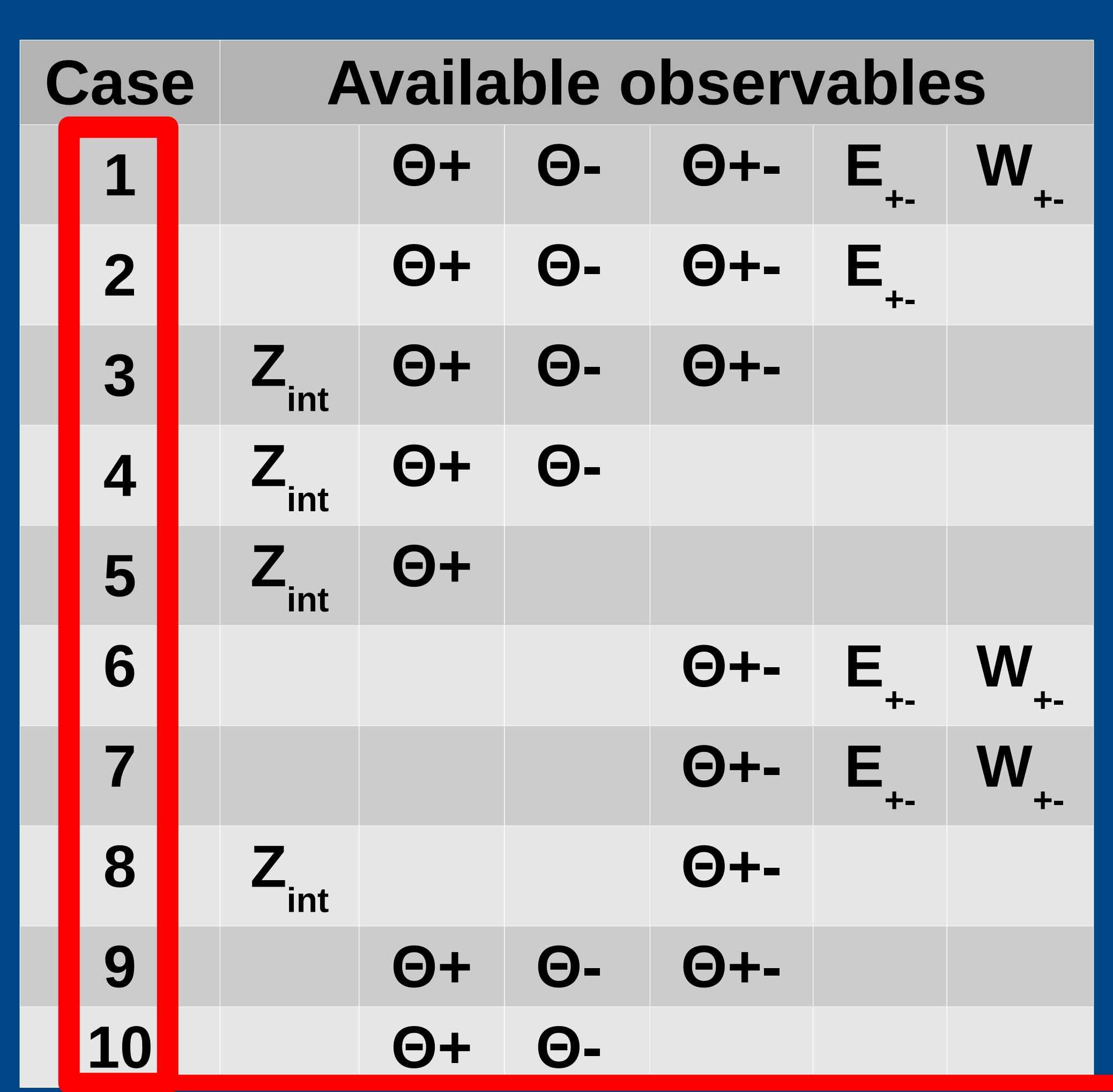

Table 2: Different combinations of observables considered
Figure 6: Invariant mass (W) distributions. (Area Normalized)
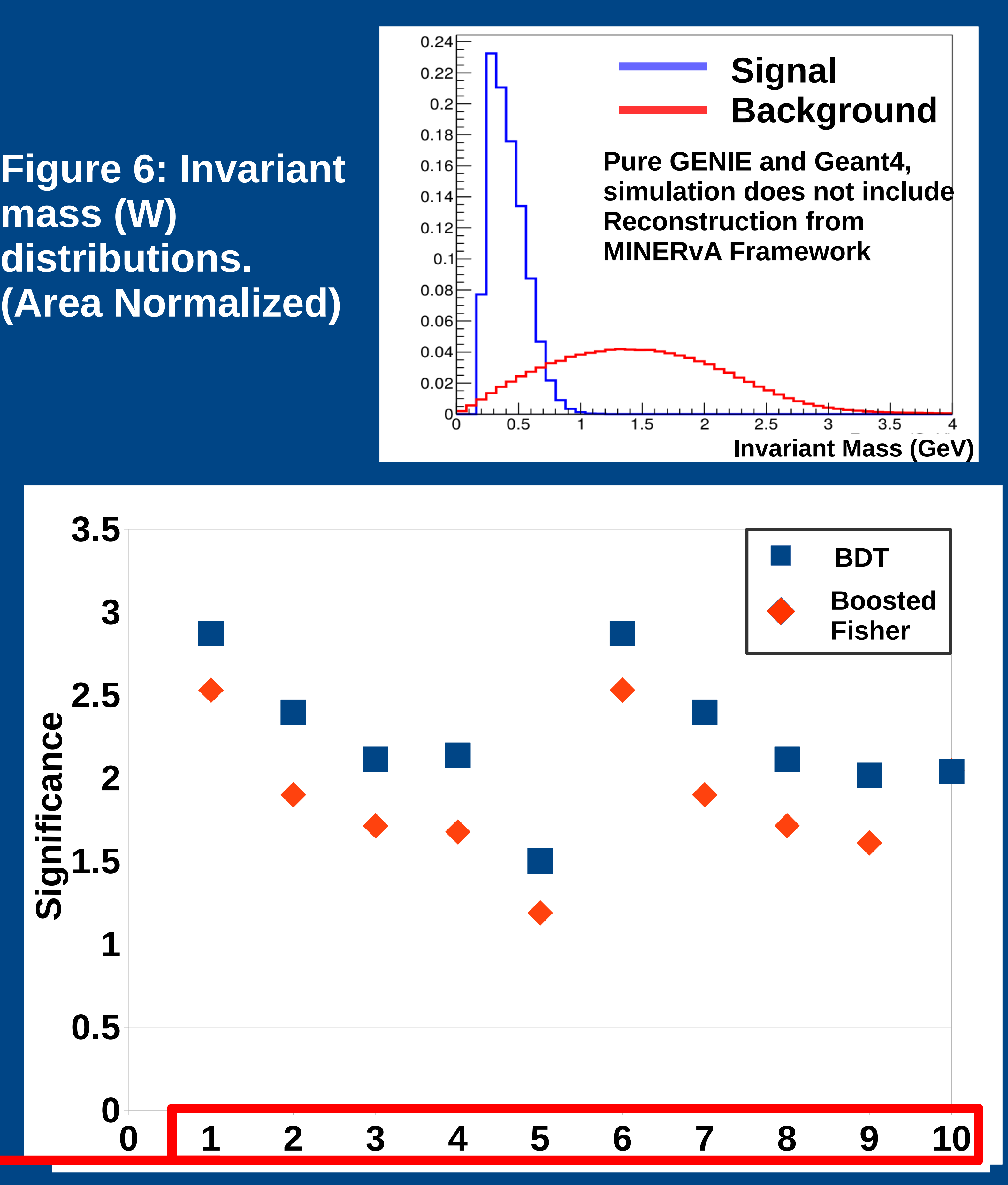

Figure 8: Significance Plot for BDT and Boosted Fisher, for the different scenarios in Table 2.

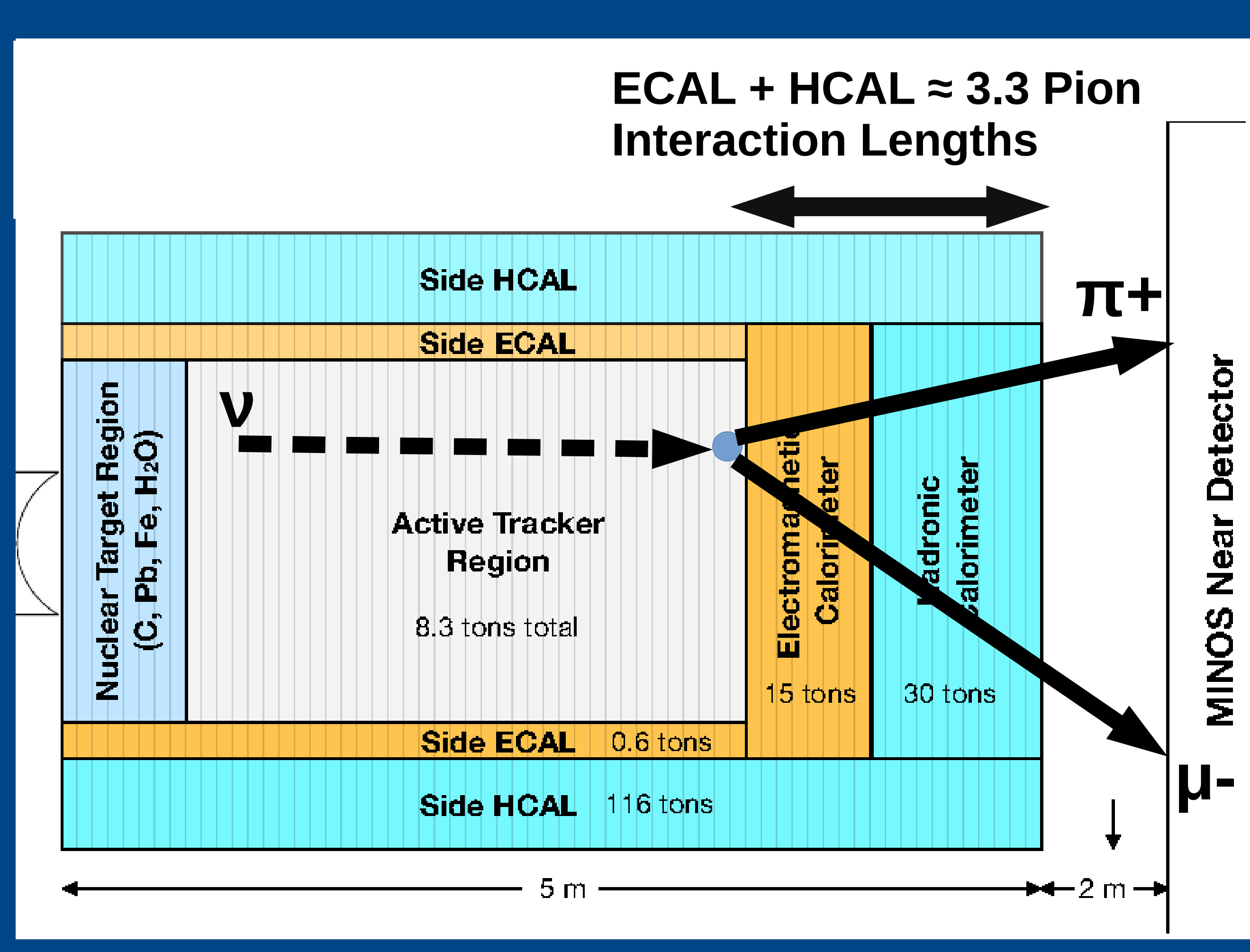

Figure 3: A $\pi+$ is produced near the frontend of the detector, escapes, and is detected as a $\mu+$ by MINOS

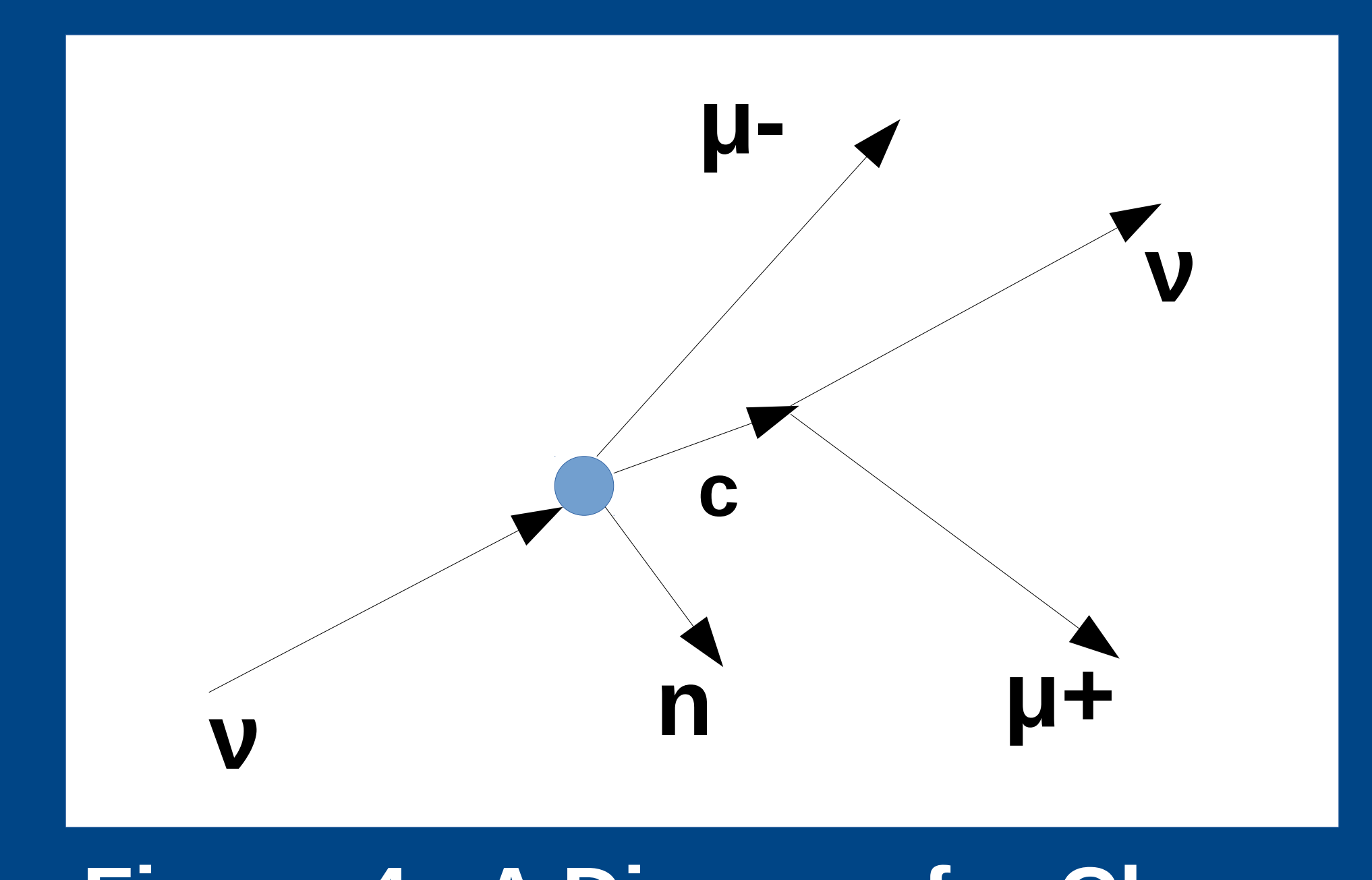

Figure 4: A Diagram for Charm Production

\section{ween $\mu+$ coming pion track} $r$

CONCLUDING REMARKS

- When compared, BDT performed better than Boosted Fisher for all of the 10 scenarios

- Invariant mass (W) as well as the angle between muons $(\Theta+-)$ proved powerful for the signal selection

\section{REFERENCES}

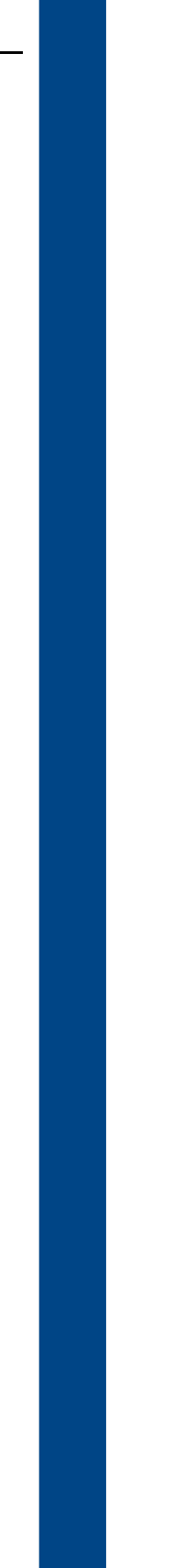

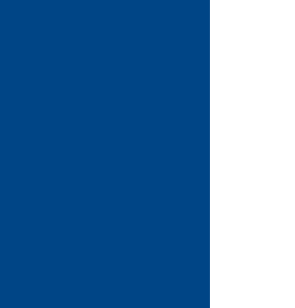

